PROCEEDINGS OF THE

AMERICAN MATHEMATICAL SOCIETY

Volume 98. Number 4. December 1986

\title{
ON A PROBLEM OF MAGNUS
}

\author{
ALEXANDER LUBOTZKY ${ }^{1}$ \\ In honor of Boris Weisfeiler
}

\begin{abstract}
By using "strong approximation" results for linear group a question of Magnus on residual properties of free groups is answered affirmatively.
\end{abstract}

Let $\mathscr{C}$ be a nonempty class of groups and $\Gamma$ a group. $\Gamma$ is said to be residually- $\mathscr{C}$ if for every $g \neq 1$ in $\Gamma$ there is an epimorphism $\phi: G \rightarrow C$ where $C$ is a group belonging to $\mathscr{C}$ s.t. $\phi(g) \neq 1$. Equivalently the intersection of all normal subgroups $N$ of $\Gamma$ for which $\Gamma / N$ belongs to $\mathscr{C}$ is the unit element of $G$.

The residual properties of the free groups $F_{n}$ received a lot of attention: They had been known to be residually finite even before $P$. Hall coined the phrase "residually finite". They are also known to be residually- $\mathscr{C}(p)$ where $\mathscr{C}(p)$ is the class of finite $p$-groups for a fixed prime $p$.

In 1968 Wilhelm Magnus surveyed the state of the art of residually finite groups and wrote [M, p. 309]:

"For $n \geqslant 2, F_{n}$ is not only residually- $\mathscr{C}(p)$ for every prime number $p$, it is also residually- $A$ and residually-PSL $\left(2, p^{k}\right)$ for fixed $k$ and variable $p$, where $A$ denotes the class of alternating groups.... Here, incidentally the story ends. It is not even known whether $F_{2}$ is residually-PSL $(3, p)$ where $p$ runs though all primes or residually-PSL $\left(2,2^{k}\right)$ where $k$ runs through the positive integers."

In this note we observe that these two questions can be answered affirmatively by using recent deep "strong approximation" theorems for linear groups proved by Weisfeiler [W] (see also Nori [N] and Matthews-Vaserstein-Weisfeiler [MVW]).

These results say, for example, that if $\Gamma$ is a subgroup of $\operatorname{SL}(3, \mathbf{Z})$ which is Zariski dense in $\operatorname{SL}(3, \mathbf{C})$, then its closure in the congruence topology of $\operatorname{SL}(3, \mathbf{Z})$ is of finite index in $\operatorname{SL}(3, \mathbf{Z})$.

In particular, for almost all primes $p, \Gamma$ is mapped onto $\operatorname{SL}(3, p)$ under the canonical map $\phi_{p}: \operatorname{SL}(3, \mathbf{Z}) \rightarrow \operatorname{SL}(3, \mathbf{Z} / p \mathbf{Z})$. The intersection of all kernels of $\phi_{p}$, for an infinite set of primes, is trivial and so $\Gamma$ is residually- $\{\operatorname{SL}(3, p) \mid p$ prime $\}$ (and also residually- $\operatorname{PSL}(3, p)$ if the center of $\Gamma$ is trivial). To answer Magnus' first question it remains now to check that $F_{2}$ can be embedded as a Zariski-dense subgroup in $\operatorname{SL}(3, \mathbf{Z})$. Indeed, $\operatorname{SL}(3, \mathbf{Z})$ is Zariski-dense in $\operatorname{SL}(3, \mathbf{C})$ by Borel Density

Received by the editors July 19, 1985 and, in revised form, November 1, 1985.

1980 Mathematics Subject Classification (1985 Revision). Primary 20E26, $20 \mathrm{H} 05$.

${ }^{1}$ This work was done while the author was visiting the University of Chicago, whose warm hospitality and support are gratefully acknowledged.

(C1986 American Mathematical Society $0002-9939 / 86 \$ 1.00+\$ .25$ per page 
Theorem [R, Chapter V] and any Zariski dense subgroup contains a Zariski-dense subgroup isomorphic to $F_{2}$ by a famous result of Tits [T, Theorem 3].

For the second case $\mathscr{C}=\left\{\operatorname{PSL}\left(2,2^{k}\right) \mid k \in \mathbf{N}\right\}$ again it suffices to prove for $\mathscr{C}=\left\{\operatorname{SL}\left(2,2^{k}\right) \mid k \in \mathbf{N}\right\}$ and we argue essentially the same way: This time use Theorem 4 of [T] to embed $F_{2}$ as a Zariski-dense subgroup of $\mathrm{SL}_{2}\left(\mathbf{F}_{2}[X]\right.$ ) (where $\mathbf{F}_{2}[X]$ is the polynomial ring over the field of order $\left.2, \mathbf{F}_{2}\right)$ and then use Theorem 8.2 of [W] to conclude that $\Gamma=F_{2}$ is mapped onto $\mathrm{SL}_{2}\left(\mathbf{F}_{2}[X] / \mathbf{M}\right)$ for almost every maximal ideal $\mathbf{M}$ of $\mathbf{F}_{2}[X]$. But $\mathbf{F}_{2}[X] / \mathbf{M} \simeq \mathbf{F}_{2^{k}}$ for some $k$. So $F_{2}$ is residually$\mathrm{SL}_{2}\left(2^{k}\right)$. This settles the second question of Magnus.

The same arguments work in a much more general content.

TheOREM. Let $G$ be a Chevalley group scheme defined over $\mathbf{Z}($ e.g., $G=\operatorname{SL}(n)$, $\operatorname{Sp}(2 n)$ etc.). Then

(a) For a fixed $k$ and variable $p, F_{2}$ is residually- $\left\{G\left(p^{k}\right) \mid p\right.$ prime $\}$.

(b) For a fixed $p$ and variable $k, F_{2}$ is residually- $\left\{G\left(p^{k}\right) \mid k \in \mathbf{N}\right\}$ unless $p=2$ and $G$ is of type $B_{n}, C_{n}$ or $F_{4}$ or $p=3$ and $G$ is of type $G_{2}$.

Here $G(l)$ denotes the $K$-rational points of $G$ over the field $K$ of order $l$.

For the proof of (a): Let $L$ be a Galois extension of $\mathbf{Q}$ of degree $k$ such that there exists infinitely many primes $p$ in $\mathbf{Z}$ which are still primes in $L$, i.e. the ideal $p \mathcal{O}$ of the ring of algebraic integers $\mathcal{O}$ of $L$ is a maximal ideal of $\mathcal{O}$. Such an $L$ does exist: For example, let $q$ be a prime such that $q \equiv 1(\bmod k)$ and let $M$ be the cyclotomic field $\mathbf{Q}[\exp (2 \pi i / q)]$. Then $\operatorname{Gal}(M / \mathbf{Q})$ is cyclic of order $q-1$, and we can find therefore a Galois extension $L / \mathbf{Q}$ of degree $k$ such that $L \subseteq M$. It suffices to check that infinitely rational primes are still primes in $M$. By [C, Theorem 10.45] this is indeed the case for every prime $p$ which is primitive $\bmod q-1$. There are infinitely many such primes by the Dirichlet theorem.

So $\mathcal{O}$ has infinitely many maximal ideals $\mathbf{M}$ for which $\mathcal{O} / \mathbf{M}$ is a field of order $p^{k}$ for some prime $p$. Now, consider $G$ as defined over $L$ and let $H=\operatorname{Res}_{L / Q}(G)$ be the Q-algebraic group obtained by "restriction of the scalars" (cf. [Z, p. 116]). So $H(\mathbf{Z})=G(\mathcal{O})$ (loc. cit.). Again, use the Borel density theorem and [T, Theorem 3] to find a Zariski dense subgroup $\Gamma$ of $H(\mathbf{Z})$ isomorphic to $F_{2}$. By [W, Theorem 9.1] (or Nori [N] or [MWV, p. 515]) $\Gamma$ is mapped onto $H(\mathbf{Z} / p \mathbf{Z})$ for almost all $p$. But $G(\mathcal{O} / \mathbf{M})=H(\mathbf{Z} / p \mathbf{Z})$ where $\mathbf{M}$ is the ideal $p \mathcal{O}$, so $\Gamma$ is mapped onto $G\left(p^{r}\right)$ for infinitely many primes $p$. The kernel is clearly trivial so $\Gamma$ is residually- $\left\{G\left(p^{r}\right) \mid p\right.$ prime).

The proof of the second part of the theorem is just the same as the proof for $\operatorname{SL}\left(2,2^{k}\right)$ except that this time we take $G\left(\mathbf{F}_{p}[X]\right)$. We have to exclude few cases in order to be able to apply Theorem 9.1 of [W]. It is very likely that the theorem holds for these cases as well.

REMARKS. (1) It is interesting to note that the proofs in [MVW and W] use the classification of the finite simple groups (while Nori's proof does not, but Nori's result covers only our needs for part (a) of the theorem and not for part (b)). So our theorem can also be considered as a by-product of the classification. 
(2) Every free group is residually- $\left\{F_{2}\right\}$ (cf. [M]), so our theorem holds for every free group. (This can also be seen from the proof.)

(3) The theorem says in particular that for fixed $n$ and $k$ and variable $p$, and for fixed $n$ and $p$ and variable $k, F_{2}$ is residually $\operatorname{PSL}\left(n, p^{k}\right)$. But, it leaves open the question whether $F_{2}$ is residually $\operatorname{PSL}\left(n, p^{r}\right)$ for $p$ and $r$ fixed and $n$ variable. One can also speculate and ask whether $F_{2}$ is residually every infinite set of finite simple groups.

ADDED IN PROOF. 1. Since this paper was written, I have learned from O. Kegel, M. Newman and S. Pride about other special cases of the Theorem in the literature. See $[1,2]$ and the references therein.

2. J. S. Wilson has answered the first question in Remark (3) affirmatively. The second was previously raised by Yu. M. Gorchakov (cf. [2]).

1. S. J. Pride, Residual properties of free groups, Pacific J. Math. 43 (1972), $725-733$.

2. V. M. Levchuk and Yu. W. Nuzlin, Structure of Ree groups, Algebra and Logic 24 (1985), 16-23.

\section{REFERENCES}

[C] H. Cohn, A classical invitation to algebraic numbers and class fields, Springer-Verlag, New York, 1978.

[M] W. Magnus, Residually finite groups, Bull. Amer. Math. Soc. 75 (1969), 306-316.

[MVW] C. R. Matthews, L. N. Vaserstein and B. Weisfeiler, Congruence properties of Zariski-dense subgroups, Proc. London Math. Soc. 48 (1984), 514-532.

$[\mathbf{N}]$ M. Nori, Subgroups of $\mathrm{SL}_{n}(\mathbf{Z})$ and $\mathrm{SL}_{n}\left(\mathbf{F}_{p}\right)$, Invent. Math. (to appear).

[R] M. S. Raghunathan, Discrete subgroups of Lie groups, Springer-Verlag, New York, 1972.

[T] J. Tits, Free subgroups in linear groups, J. Algebra 20 (1972), 250-270.

[W] B. Weisfeiler, Strong approximation for Zariski-dense subgroups of semisimple algebraic groups, Ann. of Math. (2) 120 (1984), 271-315.

[Z] R. Zimmer, Ergodic theory and semisimple groups, Birkhäuser, Boston-Basel-Stuttgart, 1984.

INSTITUTE OF MATHEMATICS, HeBREW UNIVERSITY, JERUSALEM, ISRAEL 